\title{
Port security/safety, risk analysis, and modeling
}

\author{
Tayfur Altiok
}

Published online: 9 February 2011

(C) Springer Science+Business Media, LLC 2011

Freely flowing international trade, carried predominantly by ocean-going vessels, has been a major contributor to the global prosperity experienced in the second-half of the 20th century. In the U.S. and many countries worldwide, maritime traffic in ports and waterways is a critical component of national supply chains and the backbone of many economies. However, the proximity of major seaports to urban centers and the volume and diversity of seaport activities render them vulnerable targets, where even small incidents could have crippling economic effects. Increasing important is that security operations must strike a balance between providing security and impeding the movement of cargo with the attendant economic costs, as any stoppage or appreciable slow-down in port operations impacts numerous supply chains, and extended stoppages necessitate diverting vessels to other ports, as was the case in the 2003 strike of longshoremen in the port complex of LA/LB.

Maintaining efficient port operations sometimes conflicts with port security measures addressing worldwide concerns about terrorism, drug smuggling, and crime. This volume explores a wide variety of topics at the interface of port security, safety, and port operations, with an emphasis on technical tools using mathematical modeling and risk analysis. In particular, this volume considers the general areas of port security and/or safety risk analysis, port and waterway maritime traffic analysis and modeling, cargo screening and inspection algorithms, nuclear detection issues and algorithms, analysis of container delays and costs due to cargo inspection, cost and economic trade-off of port security, accident probability modeling and decision analysis, and multi-objective trade-off in port security.

Such models can assist in analyzing the effectiveness of interdiction measures and the disruptive impact of high-consequence events in and around port complexes, as well as in assessing the effectiveness of strategies to mitigate and recover from such events. These models can further help in effectively setting up the "Rules of the Road" in ports and waterways.

T. Altiok $(\bowtie)$

Rutgers University, Piscataway, USA

e-mail: altiok@ rci.rutgers.edu 
Clearly, there have been security issues and major setbacks slowing the trade. Sea ports typically house major infrastructure such as container terminals, oil refineries, petrochemical facilities, bridges, and passenger terminals, all of which are targets and vulnerable to terrorist attacks with potentially high consequences. Furthermore, from the safety stand point, sea ports and waterways are the sites of major accidents such as collision, grounding, and ramming of vessels among others, ending up with spills and sunken debris and wrecks resulting in port closures. Agencies like the U.S. Coast Guard, vessel traffic services (VTS), maritime exchanges, and pilot associations establish and maintain a set of rules for vessel movements and facility operations in ports and waterways in which risk assessment, analysis, and management have had a major role.

The term risk is used to mean many different things in different fields of society. For instance, in the healthcare area, risk is just a probability. In the finance area, it is perceived to be variance or the deviation from the norm. In many other fields, such as the maritime domain, it also has another component that is the impact or loss. The basic method of risk analysis has been to use scenarios to describe different possible pathways to unwanted events. Each scenario has a probability of occurrence. It also has an associated consequence, which may be measured in terms of human fatalities, injuries, environmental impact, money, etc., either singly or jointly. Thus, one definition (or we should say the common definition that we support) of risk arising from an activity is that it is the collection of scenarios, probabilities, and consequences that can arise from the activity leading to the conceptual formulation of

$$
\text { risk }=\text { probability } \times \text { consequence. }
$$

This is a meaningful approach to risk since it brings both the chance and loss together. It is plausible that high-consequence events occur rarely and low-consequence events occur frequently. The meaningful measure that brings these concepts together for decision making is risk. Also, if there are different events with varying levels of risks, then the overall risk can be looked at as the sum of the risks over all the events (additive nature of risk).

Risk assessment plays an important part in ensuring compliance with regulation in many fields such as environmental protection, healthcare, transportation, food and pharmaceutical, among others. The absolute level of risk can be estimated, and the potential risk impact of different risk reduction measures can be quantified. By taking into account the costs of the risk reduction measures and their benefits, it is possible to advise the decision maker about appropriate actions. It should also be emphasized however that quantitative considerations alone should not be the deciding factor in choosing a particular risk reduction measure. Other issues such as good practice, constraints, adverse consequences, and appropriate regulatory practice are also important.

Furthermore, risk analysis and management are used by policy makers when allocating resources and taking actions under uncertainty. For this reason, developing better models and approaches for effective and improved risk analysis is of strategic interest to port authorities, terminal operators, and industrial and government agencies.

There are qualitative and quantitative approaches to risk analysis. Qualitative approaches are solely based on surveying expert opinion by using some key questions to determine what are the unwanted events, their chances of occurrence, and their consequences. For instance in the maritime domain, the U.S. Coast Guard used this approach (PAWSA-Ports And Waterways Safety Analysis) in over 40 ports to decide whether they need a VTS system or not. The drawback of such an approach is that it lacks a detailed and scientific analysis and can easily lead to seriously erroneous conclusions. 
As a remedy, quantitative models started being developed for both safety and security risks during the last decade. Especially, probabilistic risk analysis models have been developed involving conditional probabilities of events and consequences. In such an approach, one again has to think of all possible events and outcomes for which some historical data are typically available. In a more rigorous approach, a high-fidelity simulation model is built to generate all potential events (e.g., vessel collisions, groundings, ramming, spills, and others in safety related scenarios) along with a range of consequences that are passed to a mathematical risk formulation as they occur in the simulation model. In such an approach no events and consequences are missed and a risk profile of the port or the waterway is produced over time and space that will help decision makers to generate various ways to mitigate risks. An issue in all these approaches is that although the concept is generic, the models have to be developed for each port separately since the geography and characteristics of each port and waterway are unique.

Security risks involve a separate set of parameters such as threat, vulnerability, and consequence as follows:

security risk $=$ threat $\times$ vulnerability $\times$ consequence,

where threat is the probability of attack, vulnerability is the probability of success given an attack occurs, and consequence is the loss given that an attack occurs and it is successful. Clearly data requirements and availability are bigger issues in assessing security risks.

A major difference between safety and security risk management is that once a mitigation policy is in place to minimize, say, vessel collisions, it can be implemented for a long while, until drastic changes occur in the system. Security risk mitigation policies may lose their effectiveness as soon as they are understood by terrorists. Thus, security policy management is similar to games in that moves need to be changed depending on intelligence gathered about terrorists' intensions. Policies against piracy in Somalia and the surrounding region are good examples. The Strait of Hormuz is a very important waterway for the region, used for moving goods for imports and exports, and is a very good example of a region bearing such risks and vulnerabilities. Game theoretic models are being built to develop effective security risk management policies considering what terrorists may do and what the vulnerabilities are at the ports and waterways. These optimization models can be very useful, however they have inherent data and information needs that may be hard to overcome.

Thus, risk analysis and management is both an art and a science. It is progressing fast and will be highly influential in decision making in the years to come regarding global supply chain management and maritime transportation. I believe this volume will serve as an important source for researchers focusing on these issues in the years to come. 